\title{
Mesure de la permittivité complexe de liquides de référence pour la détermination du débit d'absorption spécifique (DAS)
}

\author{
Measurement of the complex permittivity \\ of reference liquids for the determination \\ of the Specific Absorption Rate (SAR)
}

\author{
Djamel ALLAL et Alexis LITWIN
}

Laboratoire national de métrologie et d'essais (LNE), 29 Avenue Roger Hennequin, 78197 Trappes Cedex, France, djamel.allal@lne.fr.

\section{Résumé}

Cet article présente nos travaux pour la mesure de la permittivité complexe de liquides, réalisés dans le cadre d'un projet européen de recherche en métrologie intitulé «Traceable measurement of field strength and SAR for the Physical Agents Directive ». Ce projet avait notamment pour but d'établir la traçabilité des mesures de débit d'absorption spécifique au SI jusqu'à $10 \mathrm{GHz}$. Nous utilisons un analyseur de réseau vectoriel pour mesurer le paramètre $S_{21}$ de cellules coaxiales contenant le liquide à caractériser (un liquide de référence ou un liquide équivalent tissu). La méthode employée ne nécessite pas que l'analyseur de réseau soit étalonné en termes d'impédance. En effet, seules la longueur des cellules coaxiales et la fréquence de travail ont besoin d'être connues.

MOTS CLÉS : MÉTROLOGIE ÉLECTRIQUE, TRAÇABILITÉ, PERMITTIVTÉ COMPLEXE, LIQUIDE DE REFERENCE, DAS.

\footnotetext{
Abstract

This paper presents our work for the measurement of the complex permittivity of liquids, carried out in the framework of a European Metrology research project entitled "Traceable measurement of field strength and SAR for the Physical Agents Directive". One of the aims of this project was to establish the traceability of the specific absorption rate measurements to the SI up to $10 \mathrm{GHz}$. We use a vector network analyser to measure the $S_{21}$ parameter of coaxial cells containing the liquid under test ( a reference liquid or an equivalent tissue liquid). The method does not require the network analyser to be calibrated in terms of impedance. In fact, only the length of the coaxial cell and the working frequency need to be known.
}

\section{Introduction}

Cet article présente nos travaux dans le domaine de la métrologie de la permittivité des liquides, réalisés dans le cadre d'un projet européen de recherche en métrologie (JRP) intitulé "Traceable measurement of field strength and SAR for the Physical Agents Directive (EMF and $S A R$ )" et répondant à la directive européenne concernant les prescriptions minimales de sécurité et de santé relatives à l'exposition des travailleurs aux risques dus aux agents physiques (champs électromagnétiques). Ce projet a notamment pour but d'établir la traçabilité des mesures de débit d'absorption spécifique (DAS ou SAR en anglais) au SI jusqu'à 10 GHz.

Les travaux présentés dans cet article concernent le développement d'une méthode de mesure de la permittivité complexe à l'aide d'un analyseur de réseau vectoriel et la réalisation de cellules destinées à la mesure de la permittivité complexe de liquides de référence et de liquides équivalents tissus ou liquides de DAS.

\section{Définitions}

Le DAS est défini comme le débit avec lequel l'énergie est absorbée par unité de masse du tissu, c'est-à-dire la puissance absorbée par unité de masse. Il s'exprime en $\mathrm{W} \cdot \mathrm{kg}^{-1}$ et peut être déterminé à partir de la connaissance du champ électrique $E$ appliqué et mesuré à l'aide 


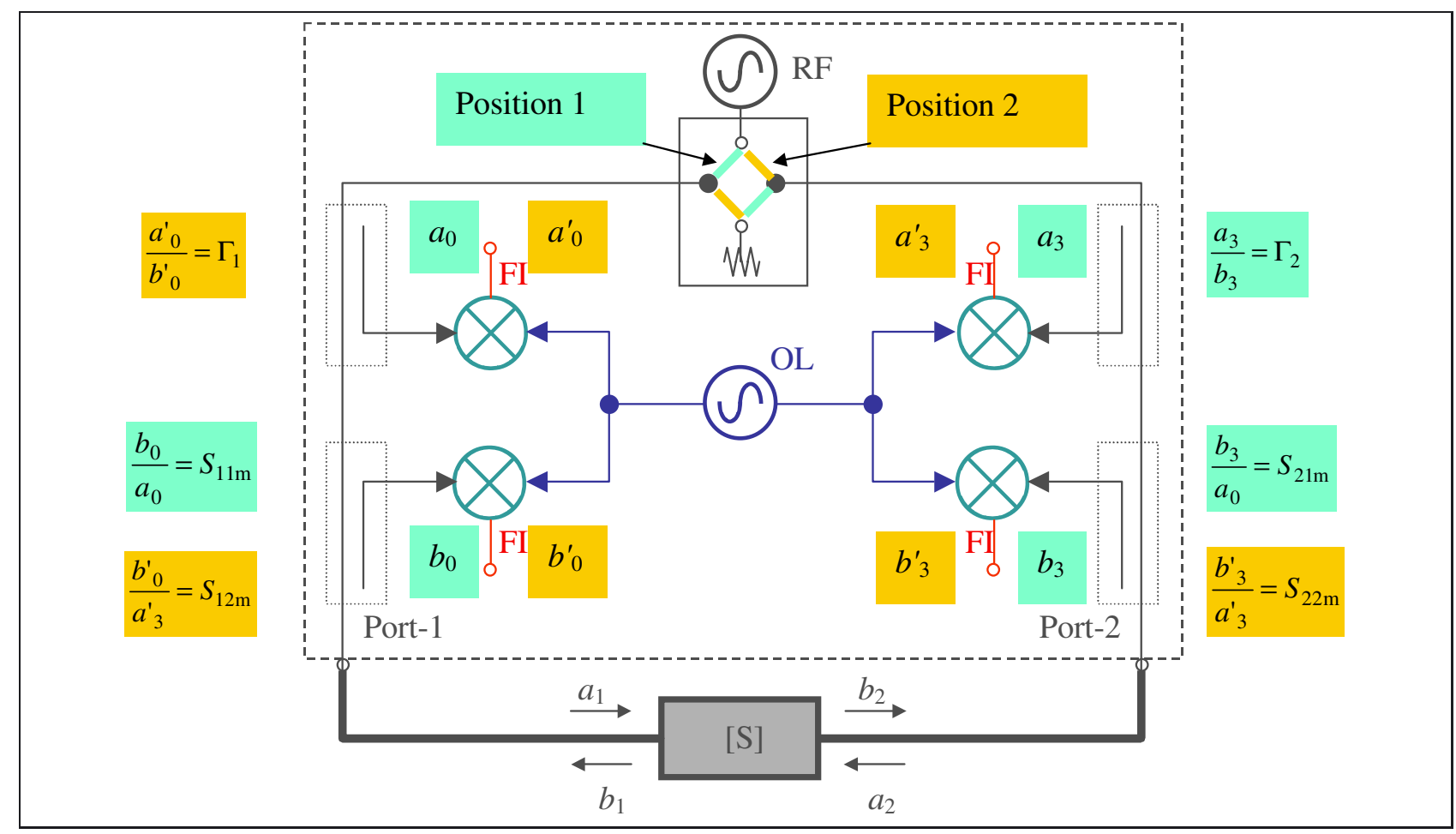

Fig. 1. - Rapports d'ondes mesurables par un analyseur de réseau à quatre récepteurs de mesure.

d'une sonde de champ électromagnétique, de la conductivité $\sigma$ et de la masse volumique $\rho$ du milieu par :

$$
\text { DAS }=\sigma \frac{E^{2}}{\rho} .
$$

La traçabilité des mesures de DAS nécessite donc de connaître le milieu à caractériser et donc sa permittivité complexe.

La permittivité d'un milieu décrit sa réponse à un champ électrique appliqué. Pour caractériser un milieu, on s'intéresse plutôt à la permittivité relative $\varepsilon_{r}$ qui est une grandeur sans unité et définie comme le rapport de la permittivité (absolue) du milieu à la permittivité du vide $\varepsilon_{0}$ (exprimées en $\left.\mathrm{F} \cdot \mathrm{m}^{-1}\right)$.

Généralement, le terme «relative » et l'indice « $\mathrm{r} »$ sont omis par commodité et sans risque d'ambiguïté.

La permittivité est généralement une grandeur complexe. Sa partie réelle $\varepsilon^{\prime}$ est liée à l'énergie stockée dans le milieu et sa partie imaginaire $\varepsilon^{\prime \prime}$ à la dissipation d'énergie (pertes) et peut s'écrire en fonction de la conductivité du milieu et de la fréquence $f$ par :

$$
\varepsilon^{\prime \prime}=\frac{\sigma}{2 \pi f \varepsilon_{0}} .
$$

\section{Méthode de mesure}

Les mesures de permittivité complexe large bande sont réalisées à l'aide d'un analyseur de réseau vectoriel. En effet, l'analyseur de réseau permet de mesurer les paramètres $S$ de lignes de transmission dont les valeurs dépendent de la permittivité complexe du diélectrique constituant ces lignes. Une procédure d'extraction de la permittivité des valeurs de paramètres $S$ est alors appliquée afin de déterminer la permittivité du milieu.

Le liquide mesuré est introduit dans une cellule coaxiale, en fait un tronçon de ligne coaxiale avec des plans de référence bien définis. Ce liquide occupe l'espace compris entre les deux conducteurs, interne et externe, de la ligne coaxiale et joue le rôle du diélectrique de cette ligne.

La méthode de mesure employée ne nécessite pas l'étalonnage de l'analyseur de réseau (en termes d'impédance). En effet, la constante de propagation dans la ligne de transmission peut être déterminée simplement à partir de la mesure de rapports d'ondes entrantes et sortantes, c'est-à-dire ce qui est communément désigné par paramètres $S$ bruts ou non corrigés [1]. Cette méthode, appelée méthode TL (Through-Line), est en fait une des étapes de la méthode d'étalonnage TRL (ThroughReflect-Line) [2]. Ici, la ligne «Line » est une cellule remplie de liquide.

En effet, si l'on considère le modèle d'analyseur de réseau à quatre récepteurs de mesure de la figure 1 [3] et en négligeant les effets de crosstalk, un modèle à huit termes d'erreur peut être appliqué. Par ailleurs, le fait de disposer de quatre récepteurs de mesure permet de mesurer, en plus des quatre paramètres $S_{i j \mathrm{~m}}$, les facteurs de réflexion des ports 1 et 2 de l'analyseur de réseau, respectivement $\Gamma_{1}$ (position 2) et $\Gamma_{2}$ (position 1$)$.

Ces six paramètres sont définis sur la figure 1.

L'indice «m» indique qu'il s'agit ici des paramètres $S$ bruts mesurés, non corrigés (c'est-à-dire sans 
détermination des termes d'erreur à partir de la mesure d'étalons).

Le commutateur étant en position 1, la relation entre les ondes entrantes et les ondes sortantes mesurées, peut être donnée par :

$$
\left(\begin{array}{l}
b_{0} \\
a_{0}
\end{array}\right)=\mathbf{X} \cdot \mathbf{T} \cdot \mathbf{Y} \cdot\left(\begin{array}{l}
a_{3} \\
b_{3}
\end{array}\right)
$$

où $\mathbf{X}$ et $\mathbf{Y}$ représentent respectivement les deux matrices d'erreur des réflectomètres 1 et 2 et $\mathbf{T}$ la matrice des paramètres $T$ du quadripôle à caractériser.

De la même manière, quand le commutateur est en position 2 :

$$
\left(\begin{array}{l}
b_{0}^{\prime} \\
a_{0}^{\prime}
\end{array}\right)=\mathbf{X} \cdot \mathbf{T} \cdot \mathbf{Y} \cdot\left(\begin{array}{l}
a_{3}^{\prime} \\
b_{3}^{\prime}
\end{array}\right)
$$

La matrice $\mathbf{T}$ permet d'écrire les ondes à l'entrée en fonction des ondes en sortie du quadripôle sous test et elle peut s'écrire en fonction des paramètres $S$ :

$$
\mathbf{T}=\left(\begin{array}{ll}
T_{11} & T_{12} \\
T_{21} & T_{22}
\end{array}\right)=\frac{1}{S_{21}} \times\left(\begin{array}{cc}
-S_{11} S_{22}+S_{12} S_{21} & S_{11} \\
-S_{22} & 1
\end{array}\right) .
$$

Nous définissons la matrice $\mathbf{M}$ dite matrice de mesure :

$$
\mathbf{M}=\left(\begin{array}{ll}
b_{0} & b_{0}^{\prime} \\
a_{0} & a_{0}^{\prime}
\end{array}\right) \times\left(\begin{array}{ll}
a_{3} & a_{3}^{\prime} \\
b_{3} & b_{3}^{\prime}
\end{array}\right)^{-1}=\mathbf{X} \cdot \mathbf{T} \cdot \mathbf{Y} .
$$

Le développement de cette relation matricielle permet de mettre en évidence six rapports d'ondes, dont les quatre paramètres $S_{i j \mathrm{~m}}$ et les deux rapports $\frac{a_{3}}{a_{0}}$ et $\frac{a_{0}^{\prime}}{a_{3}^{\prime}}$ entre les ondes incidentes respectivement des ports 1 et 2 quand le commutateur est en position 1 puis 2 :

$$
\mathbf{M}=\left(\begin{array}{cc}
\frac{\frac{b_{3}}{a_{0}} \frac{b_{0}^{\prime}}{a_{3}^{\prime}}-\frac{b_{0}}{a_{0}} \frac{b_{3}^{\prime}}{a_{3}^{\prime}}}{\frac{b_{3}}{a_{0}}-\frac{a_{3}}{a_{0}} \frac{b_{3}^{\prime}}{a_{3}^{\prime}}} \frac{\frac{a_{3}}{a_{0}}-\frac{b_{0}^{\prime}}{a_{0}} \frac{b_{3}}{a_{0}}-\frac{a_{3}}{a_{0}} \frac{b_{3}^{\prime}}{a_{3}^{\prime}}}{-\frac{b_{3}^{\prime}}{a_{3}^{\prime}}+\frac{b_{3}}{a_{0}} \frac{a_{0}^{\prime}}{a_{3}^{\prime}}} & 1-\frac{a_{3}}{a_{0}} \frac{a_{0}^{\prime}}{a_{3}^{\prime}} \\
\frac{b_{3}}{a_{0}}-\frac{a_{3}}{a_{0}} \frac{b_{3}^{\prime}}{a_{3}^{\prime}} & \frac{b_{3}}{a_{0}}-\frac{a_{3}}{a_{0}} \frac{b_{3}^{\prime}}{a_{3}^{\prime}}
\end{array}\right) .
$$

$\mathrm{Si}$ on remarque que :

$$
\begin{aligned}
& \frac{a_{3}}{a_{0}}=\frac{a_{3}}{b_{3}} \times \frac{b_{3}}{a_{0}}=S_{21 \mathrm{~m}} \cdot \Gamma_{2} \\
& \frac{a_{0}^{\prime}}{a_{3}^{\prime}}=\frac{a_{0}^{\prime}}{b_{0}^{\prime}} \times \frac{b_{0}^{\prime}}{a_{3}^{\prime}}=S_{12 \mathrm{~m}} \Gamma_{1}
\end{aligned}
$$

alors on peut écrire la matrice de mesure de la manière suivante :

$$
\mathbf{M}=\left(\begin{array}{ll}
\frac{S_{21 \mathrm{~m}} S_{12 \mathrm{~m}}-S_{11 \mathrm{~m}} S_{22 \mathrm{~m}}}{S_{21 \mathrm{~m}}\left(1-S_{22 \mathrm{~m}} \Gamma_{2}\right)} & \frac{S_{11 \mathrm{~m}}-S_{12 \mathrm{~m}} S_{21 \mathrm{~m}} \Gamma_{2}}{S_{21 \mathrm{~m}}\left(1-S_{22 \mathrm{~m}} \Gamma_{2}\right)} \\
\frac{-S_{22 \mathrm{~m}}+S_{12 \mathrm{~m}} S_{21 \mathrm{~m}} \Gamma_{1}}{S_{21 \mathrm{~m}}\left(1-S_{22 \mathrm{~m}} \Gamma_{2}\right)} & \frac{1-S_{12 \mathrm{~m}} S_{21 \mathrm{~m}} \Gamma_{2} \Gamma_{1}}{S_{21 \mathrm{~m}}\left(1-S_{22 \mathrm{~m}} \Gamma_{2}\right)}
\end{array}\right) .
$$

L'extraction de la permittivité complexe du liquide qui est introduit dans la cellule peut alors être effectuée à partir de deux mesures «brutes », effectuées sans correction :

- la mesure de la connexion directe entre les deux ports de l'analyseur de réseau ;

- la mesure d'une cellule remplie de liquide.

En effet, la connexion directe donne la matrice de mesure $\mathbf{M}_{\mathbf{T}}$ :

$$
\mathbf{M}_{\mathbf{T}}=\mathbf{X} \cdot \mathbf{T}_{\mathrm{T}} \cdot \mathbf{Y}=\mathbf{X} \cdot\left(\begin{array}{ll}
1 & 0 \\
0 & 1
\end{array}\right) \cdot \mathbf{Y}=\mathbf{X} \cdot \mathbf{Y}
$$

et la cellule contenant le liquide donne la matrice de mesure $\mathbf{M}_{\mathbf{L}}$ :

$$
\mathbf{M}_{\mathbf{L}}=\mathbf{X} \cdot \mathbf{T}_{\mathrm{L}} \cdot \mathbf{Y}=\mathbf{X} \cdot\left(\begin{array}{cc}
e^{-\gamma L} & 0 \\
0 & e^{+\gamma L}
\end{array}\right) \cdot \mathbf{Y}
$$

où $\gamma$ est la constante de propagation dans la cellule et $L$ la longueur de la cellule.

Cette description de la matrice $\mathbf{T}_{\mathbf{L}}$ est correcte si l'on considère comme impédance de référence, l'impédance caractéristique de la cellule [4]. En effet, la cellule constitue une ligne de transmission homogène qui est toujours adaptée à son impédance caractéristique $\left(S_{11}=S_{22}=0\right)$ ou $\left(T_{12}=T_{21}=0\right)$.

On peut alors, à partir de la relation (13) :

$$
\begin{aligned}
\mathbf{M}_{\mathbf{L}} \cdot \mathbf{M}_{\mathbf{T}}^{-\mathbf{1}} & =\mathbf{X} \cdot \mathbf{T}_{\mathbf{L}} \cdot \mathbf{Y} \cdot \mathbf{Y}^{-\mathbf{1}} \cdot \mathbf{X}^{-\mathbf{1}} \\
& =\mathbf{X} \cdot \mathbf{T}_{\mathbf{L}} \cdot \mathbf{X}^{-\mathbf{1}} \\
& =\mathbf{X} \cdot\left(\begin{array}{cc}
e^{-\gamma L} & 0 \\
0 & e^{+\gamma L}
\end{array}\right) \cdot \mathbf{X}^{-\mathbf{1}}
\end{aligned}
$$

utiliser l'égalité entre traces de matrices semblables :

$$
\delta=\operatorname{trace}\left(\mathbf{M}_{\mathbf{L}} \cdot \mathbf{M}_{\mathbf{T}}^{-\mathbf{1}}\right)=\operatorname{trace}\left(\mathbf{T}_{\mathbf{L}}\right)=e^{-\gamma L}+e^{+\gamma L} .
$$

Connaissant la longueur $L$, on peut déterminer $\gamma$ à partir de la relation (15):

$$
\exp (-\gamma L)=\frac{\delta}{2} \pm \sqrt{\frac{\delta^{2}}{4}-1}
$$

Le choix de la solution est évident car $|\exp (-\gamma L)|$ doit être inférieur à 1.

La permittivité complexe, $\varepsilon$, en supposant une propagation TEM dans le cas d'une cellule coaxiale, peut alors être déduite par la relation (16) d'après [1] :

$$
\varepsilon=\left(\frac{\gamma \cdot c}{2 \pi f}\right)^{2}
$$

où $c$ est la vitesse de la lumière dans le vide et $f$, la fréquence du signal.

Si cette méthode est appliquée avec un analyseur de réseau étalonné et référencé par exemple à une impédance $Z_{\text {ref }}=50 \Omega$, alors

- la matrice $\mathbf{T}_{\mathbf{T}}$ reste toujours égale à la matrice identité (quadripôle de longueur nulle, toujours adapté à la ligne dans laquelle il est inséré); 


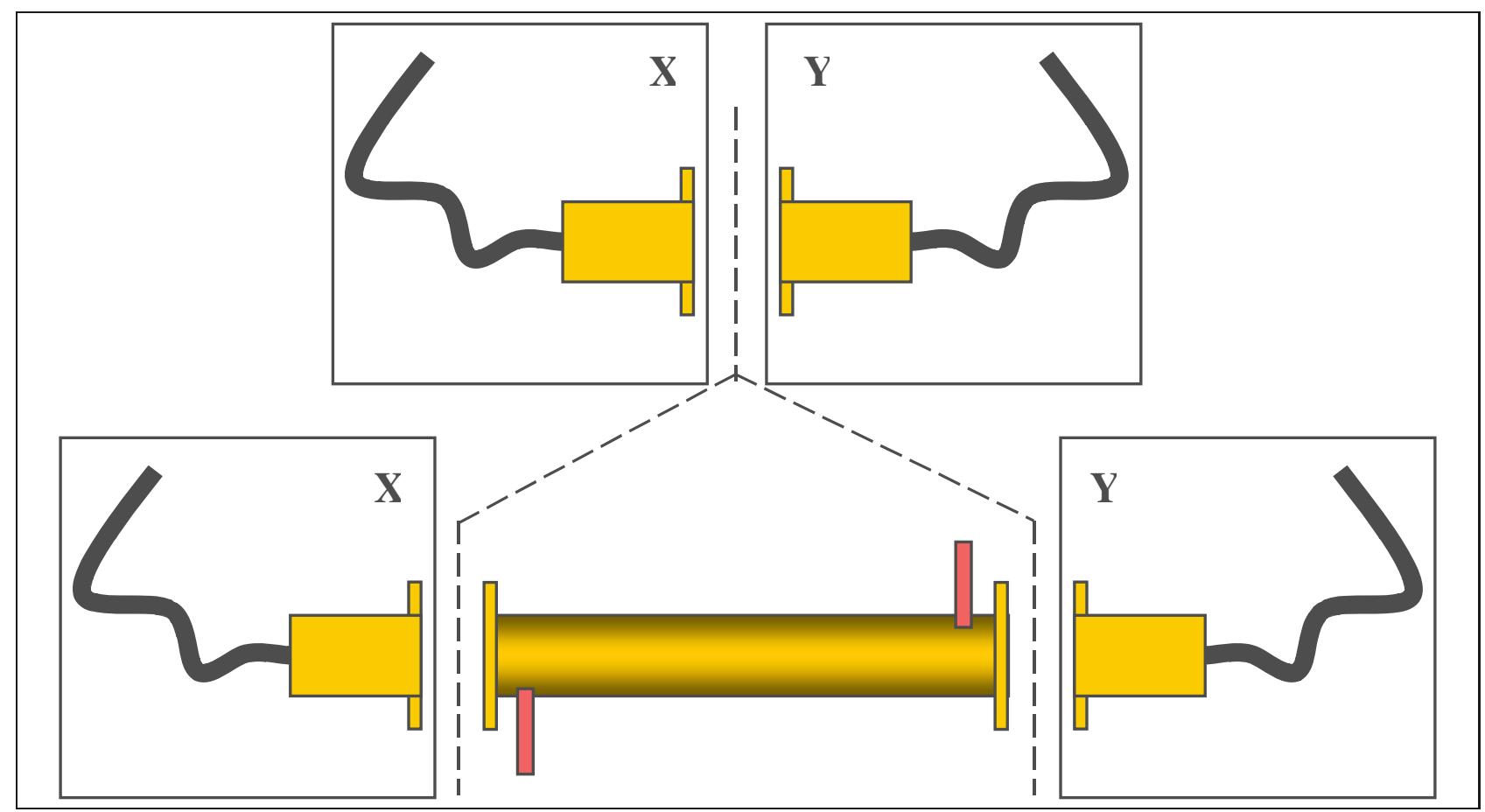

Fig. 2. - Etapes de mesure pour la méthode de détermination TL.

- et la matrice $\mathbf{T}_{\mathbf{L}}$ est décrite dans [4] comme :

$$
\mathbf{T}_{\mathrm{L}}=\frac{1}{e^{-\gamma L}\left(1-\rho^{2}\right)}\left(\begin{array}{cc}
e^{-2 \gamma L}-\rho^{2} & \rho\left(1-e^{-2 \gamma L}\right) \\
-\rho\left(1-e^{-2 \gamma L}\right) & 1-\rho^{2} e^{-2 \gamma L}
\end{array}\right)
$$

où $\rho$ peut être assimilé au facteur de réflexion de l'impédance caractéristique de la cellule par rapport à l'impédance de référence de l'analyseur de réseau et est donné par :

$$
\rho=\frac{Z_{c}-Z_{\text {ref }}}{Z_{c}+Z_{\text {ref }}}
$$

Or on peut voir que, dans ce cas, la trace de la matrice $\mathbf{T}_{\mathbf{L}}$ reste toujours égale à $e^{-\gamma L}+e^{+\gamma L}$. Donc, comme il n'est pas nécessaire de connaître $Z_{c}$, il n' est pas non plus nécessaire d'étalonner l'analyseur de réseau en termes d'impédance pour déterminer la constante de propagation et donc la permittivité complexe.

La méthode TL (fig. 2) est appliquée en utilisant une cellule pour un liquide donné et pour une bande de fréquences donnée. En effet, en fonction de la permittivité mesurée, la longueur électrique d'une même cellule et donc la variation de la constante de propagation en fonction de la fréquence peut être plus ou moins importante. Il faut donc choisir la longueur qui permet de déterminer sans équivoque la constante de propagation à partir de l'équation (15).

Si la permittivité du liquide et la fréquence de travail exigent une longueur de cellule de mesure trop petite, c'est-à-dire difficilement réalisable, il est possible d'utiliser la méthode LL, variante de la méthode TL, qui remplace la mesure de la connexion directe entre les deux ports de l'analyseur de réseau par la mesure sur une seconde cellule, de longueur différente.
Ainsi, comme montré sur la figure 3 , le plan de référence de la mesure est situé au milieu de la cellule la plus courte et les ports de l'analyseur de réseau incluent chacun une moitié de cette cellule. La méthode LL se ramène donc à une méthode TL où la cellule mesurée est une portion homogène de la cellule la plus longue, et qui a pour longueur $\Delta L$, la différence de longueur entre les deux cellules.

Les équations du système sont donc les mêmes équations que celles déjà développées, en remplaçant simplement la longueur de la cellule $L$ par la différence de longueur $\Delta L$ entre les deux cellules.

\section{Choix des liquides de référence et liquides de DAS}

Une liste de liquides de référence a été publiée par le NPL en 2001 et mise à jour en 2009 sous la référence NPL report MAT 23 [5]. Ce rapport comporte des tableaux de permittivité complexe de liquides de référence déterminée jusqu'à $5 \mathrm{GHz}$.

Ces liquides sont des liquides à molécules polaires, en général des alcools, pour lesquels les données de mesure s'accordent bien avec les paramètres de relaxation tels que ceux des modèles de Debye, simple ou double, qui permettent de décrire la variation des parties réelle et imaginaire de la permittivité complexe en fonction de la fréquence.

Ces liquides sont le butan-1-ol, le diméthylsulfoxide, l'éthanediol, l'éthanol, le méthanol, le propan-1-ol et le propan-2-ol. 


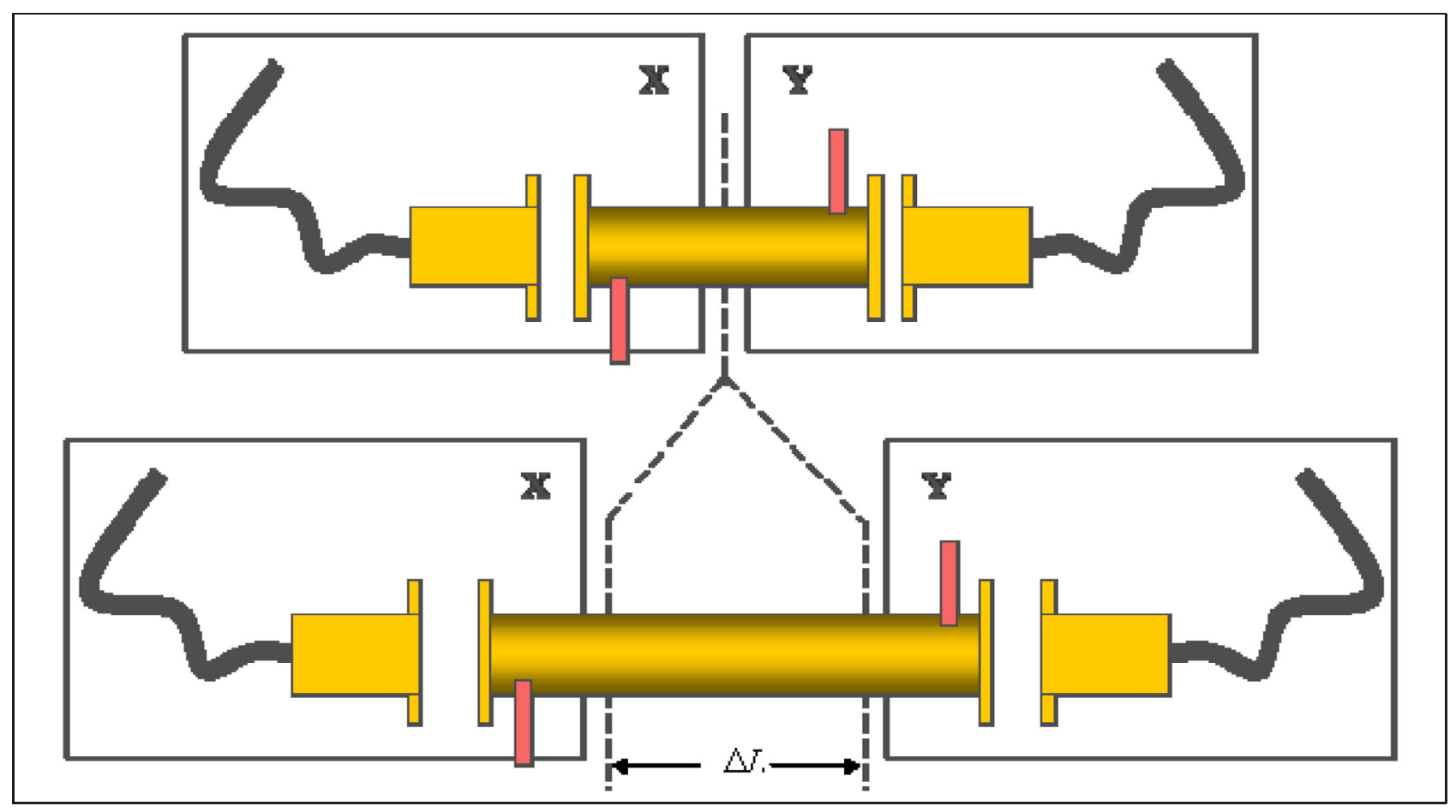

Fig. 3. - Les deux configurations successives de mesure pour la méthode de détermination LL.

Pour le travail décrit dans cet article, nous avons retenu deux liquides de cette liste, à savoir l'éthanol et le diméthylsulfoxide, pour leurs caractéristiques électromagnétiques du même ordre de grandeur que les valeurs publiées sur les tissus humains, pour la validité du modèle de relaxation qui leur est appliqué et pour des raisons pratiques telles que le coût et la disponibilité au laboratoire.

Quant aux liquides équivalents tissus ou liquides de DAS, des mélanges à base de polysorbate 20 , polysorbate 80 , polysorbate 85 (au-delà de $6 \mathrm{GHz}$ ), d'eau et de sel (chlorure de sodium), ont été proposés dans le cadre du projet européen JRP EMF\&SAR. L'un des objectifs de ce projet était la définition de mélanges, si possible non toxiques et utilisables au-delà de $6 \mathrm{GHz}$ car les normes relatives à la question des limites d'exposition ou à la mesure de l'exposition ne couvrent pas les fréquences supérieures à $6 \mathrm{GHz}$.

\section{Réalisation de cellules pour la mesure de la permittivité de liquides de référence}

Les données du rapport MAT 23 [5] s'appuient sur des mesures réalisées à l'aide de cellules en réflexion et en transmission sur ligne coaxiale $14 \mathrm{~mm}$, dotées de connecteur GR900, limitant les mesures à la fréquence maximale de $6 \mathrm{GHz}$. Cette limitation est due d'une part à la performance du connecteur GR900 qui se dégrade de manière significative aux hautes fréquences et d'autre part à la fréquence maximale d'utilisation de la ligne coaxiale $14 \mathrm{~mm}$ qui est $8,5 \mathrm{GHz}$.
Dans le but d'étendre la gamme de fréquences de mesure au-delà de $5 \mathrm{GHz}$, nous avons développé des cellules de mesure de permittivité à partir de lignes coaxiales $7 \mathrm{~mm}$, de plus petite section donc.

La ligne coaxiale $7 \mathrm{~mm}$ est conçue pour une utilisation jusqu'à $18 \mathrm{GHz}$ avec l'air comme diélectrique où seule la propagation en mode TEM est assurée. Cependant, les liquides à caractériser ont des permittivités relativement élevées par rapport à celle de l'air, ce qui abaisse nécessairement la fréquence limite d'utilisation. Nous supposons cependant que seul le mode TEM est supporté jusqu'à $10 \mathrm{GHz}$ si le matériau diélectrique utilisé est un liquide homogène et si la symétrie de la structure est assurée.

Des cellules de différentes longueurs ont été fabriquées (fig. 4). Elles disposent, à chaque extrémité du tronçon de ligne, de deux tubes d'accès afin de faire circuler un liquide thermorégulé dans la cellule. Le diamètre de ces tubes est en principe assez faible afin de ne pas perturber la propagation des ondes électromagnétiques dans la cellule pour les fréquences considérées. Des connecteurs APC7 spéciaux ont été utilisés pour réaliser l'adaptateur entre la ligne coaxiale de l'analyseur de réseau et la cellule coaxiale.

\section{Réalisation de cellules pour la mesure de la permittivité de liquides de DAS}

En deuxième étape, nous considérons la mesure de la permittivité complexe de liquides équivalents tissus ou liquides de DAS. Ces liquides peuvent présenter une 


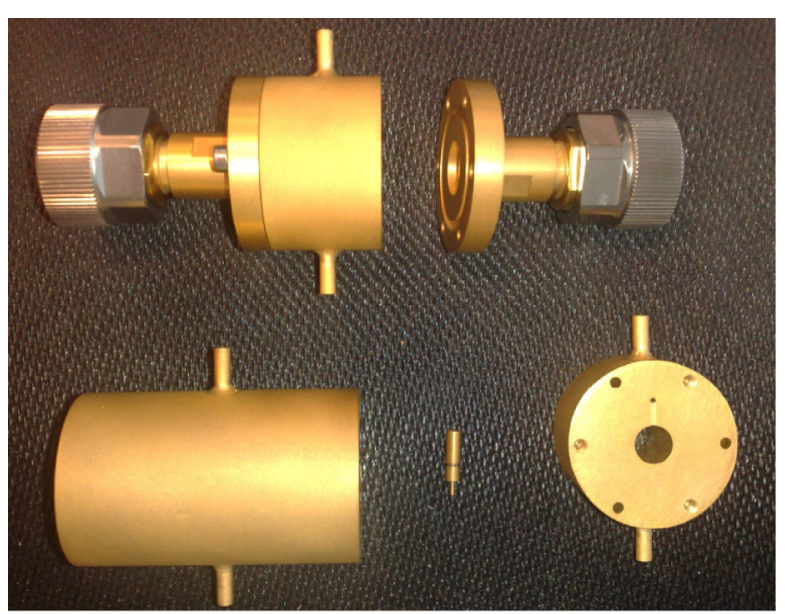

Fig. 4. - Cellules du LNE réalisées pour la mesure des liquides de référence.

viscosité importante qui empêche d'utiliser les petits tubes pour le remplissage de la cellule.

Généralement, le moyen de mesure utilisé dans ce cas est une sonde ouverte que l'on applique à la surface du liquide à caractériser par des mesures en réflexion. Afin d'extraire la caractéristique recherchée, des mesures complémentaires sont nécessaires, généralement la mesure sur un second liquide (le liquide de référence) et la mesure sur un court-circuit. L'exactitude d'une telle méthode dépend fortement de l'exactitude du modèle de couplage entre la sonde et le liquide et du modèle de court-circuit réalisé en appliquant un plan métallique sur le bout de la sonde [6].

Nous avons préféré nous tourner vers une solution plus simple à mettre en place, en restant sur la méthode de mesure décrite dans cet article, et en adaptant la conception des cellules à ce type de liquide. De plus, ceci nous permet de réduire d'un étage la chaîne de raccordement, puisque le liquide de référence n'est alors utilisé que pour la validation de la cellule de test. La permittivité du liquide de DAS est déterminée directement, au même niveau métrologique que le liquide de référence.

L'adaptation de la conception des cellules à ces liquides revient à résoudre le problème du remplissage de la cellule. Ce problème peut être résolu si le liquide est introduit avant fermeture de la cellule et si l'on s'assure qu' aucun bulle d'air n'a été emprisonnée après fermeture. La solution retenue est de fermer la cellule dans le liquide. De nouvelles cellules dont l'une des ouvertures se situe au fond d'une cuve ont donc été réalisées (fig. 5). Ainsi, le liquide est versé dans la cuve, ce qui remplit la cellule. La fermeture de la cellule, à l'aide de l'adaptateur, est alors réalisée lorsque la cuve est remplie. L'adaptateur est introduit dans le liquide à l'oblique tout en s'assurant de l'absence de bulles. Pour ce type de cellule, il est nécessaire d'utiliser un moyen externe pour la thermorégulation du liquide, comme par exemple un système à base de cellules Peltier.

Différentes longueurs de cellules ont été réalisées : $3 \mathrm{~mm}, 4 \mathrm{~mm}, 6 \mathrm{~mm}, 10 \mathrm{~mm}, 20 \mathrm{~mm}$ et $50 \mathrm{~mm}$.

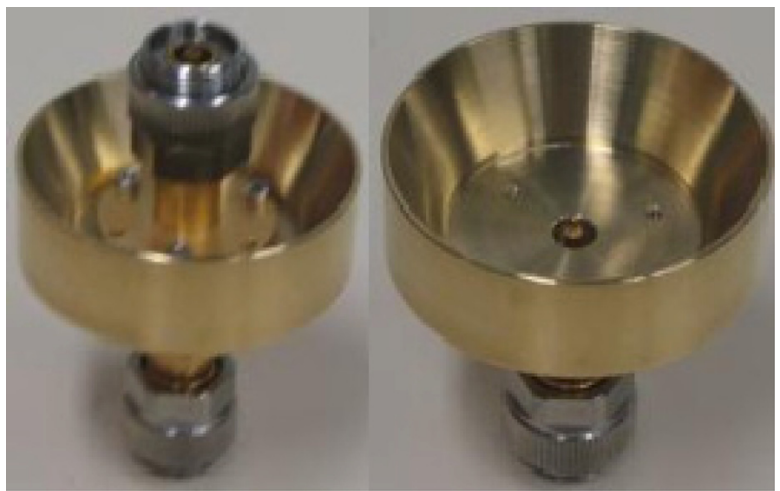

Fig. 5. - Cellules réalisées pour la mesure de liquides de DAS et liquides de référence.

Ces cellules peuvent être utilisées à la fois pour les liquides équivalents tissus et pour les liquides de référence.

\section{Mesures et détermination de la permittivité complexe de liquides}

Pour les deux types de cellules et pour les deux types de liquides, les deux variantes de la technique de mesure de la constante de propagation décrites peuvent être appliquées :

- Dans le cas de la technique TL (Through-Line), on effectue une mesure sur une cellule et on fait également la mesure sur la connexion directe entre les deux connecteurs de l'analyseur de réseau.

- Dans le cas de la technique LL (Line-Line), on fait la mesure sur deux lignes de longueurs différentes.

\section{Résultats de mesure}

En principe, le système coaxial permet de couvrir toutes les fréquences depuis la limite basse imposée par l'analyseur de réseau utilisé pour les mesures $(45 \mathrm{MHz}$ dans notre cas.). Les résultats sont montrés pour l'éthanol et le diméthylsulfoxyde, respectivement sur les figures 6 et 7 .

Pour l'éthanol, les longueurs de cellule utilisées sont $10 \mathrm{~mm}$ et $50 \mathrm{~mm}$. Les résultats sont présentés pour la gamme de $50 \mathrm{MHz}$ à $10 \mathrm{GHz}$. Pour le diméthylsulfoxyde, les longueurs de cellule utilisées sont $3 \mathrm{~mm}$ et $10 \mathrm{~mm}$. Les résultats sont montrés pour la gamme de $2 \mathrm{GHz}$ à $10 \mathrm{GHz}$. En dessous de $2 \mathrm{GHz}$, les résultats sont inexploitables. Les courbes présentent des oscillations et s'écartent fortement de la réponse attendue.

D'autres longueurs de cellule, ou combinaisons de longueurs, devraient être explorées pour obtenir des résultats plus fiables.

Les résultats du LNE sont comparés à ceux publiés par le NPL dans le rapport NPL MAT23 [5], à une température de $25^{\circ} \mathrm{C}$. 

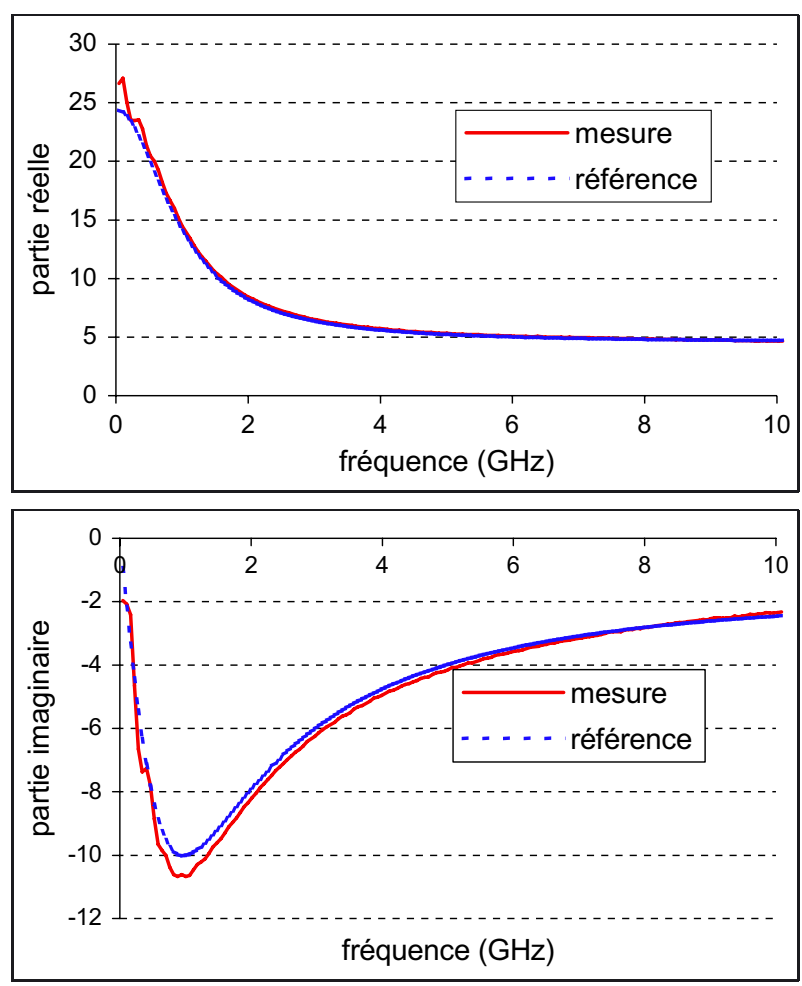

Fig. 6. - Résultats de la détermination de la permittivité complexe de l'éthanol.
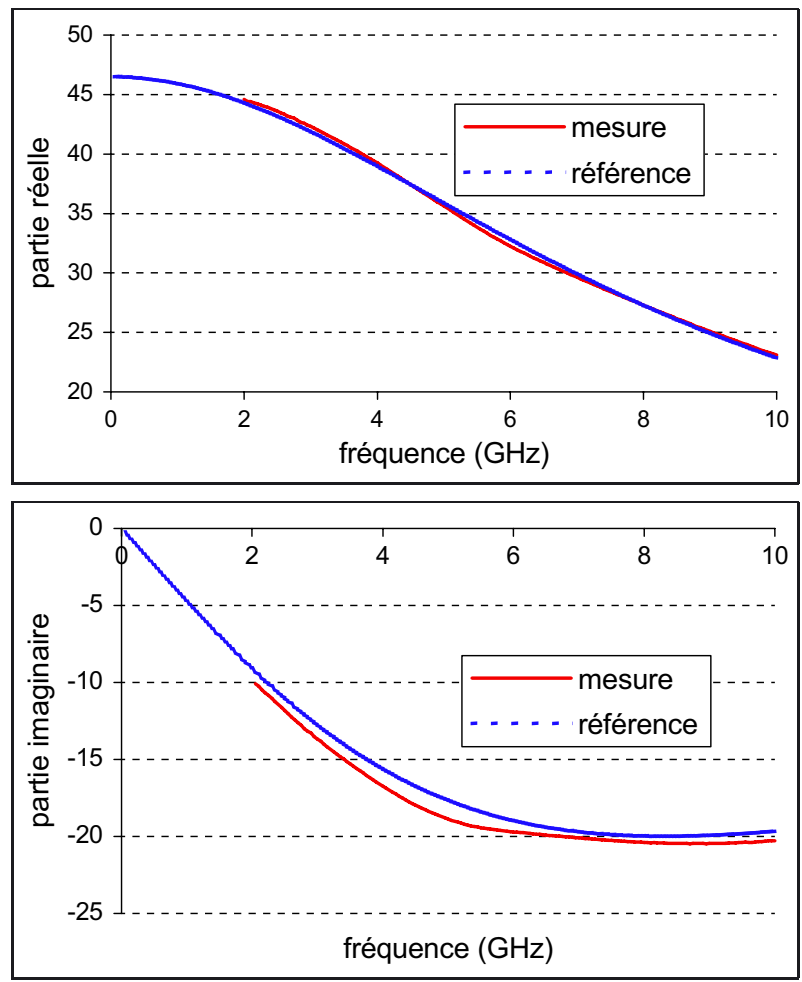

Fig. 7. - Résultats de la détermination de la permittivité complexe du diméthylsulfoxyde.

Article reçu le 20 février 2013, version révisée reçue le 11 septembre 2013.
Les valeurs de la permittivité complexe sont plus cohérentes pour la partie réelle que pour la partie imaginaire. Cet écart est plus important dans la gamme de fréquences liée à la relaxation diélectrique pour les deux liquides (cette gamme étant plus large pour le diméthylsulfoxyde).

La prise en compte des pertes métalliques abaisse légèrement la valeur absolue de la partie imaginaire de la permittivité complexe du liquide.

En outre, le système de régulation thermique de la cellule n'était pas opérationnel au moment où les mesures ont été effectuées. Compte tenu des conditions de laboratoire (régulé à $23^{\circ} \mathrm{C}$ ) et de l'échauffement de la cellule par conduction via les câbles et en raison des signaux issus de l'analyseur de réseau, la température du liquide devait être légèrement supérieure à $23^{\circ} \mathrm{C}$.

\section{Conclusion}

Dans le cadre du projet européen JRP «EMF and SAR », nous avons mis en place une méthode de mesure de la permittivité complexe à l'aide d'un analyseur de réseau vectoriel ne nécessitant pas l'étalonnage en termes d'impédance. Nous avons réalisé des cellules d'abord destinées uniquement à la mesure de permittivité de liquides de référence, puis d'autres cellules capables de mesurer à la fois celle des liquides de référence et des liquides de DAS avec la même performance métrologique. Finalement, ces moyens permettent de faire des mesures de permittivité complexe sur une large bande de fréquences, s'étendant de $500 \mathrm{MHz}$ à $10 \mathrm{GHz}$, et établissant la traçabilité de mesure de DAS pour des liquides.

\section{Références}

[1] ROLFES I., "Obstacle-based self-calibration techniques for the determination of the permittivity of liquids", $A d v$. Radio Sci., 5, 2007, 29-35.

[2] ENGEN G.F. et HoER C.A., "Thru-reflect-line: An improved technique for calibrating the dual six-port automatic network analyzer", IEEE Trans. Microwave Theory Tech., 27, 12, 1979, 987-993.

[3] RYTTING D., "Network analyzer error models and calibration methods", 54th ARFTG Conference short notes, déc. 2000 .

[4] EUL H.J. et SCHIEK B., "A generalized theory and new calibration procedures for network analyzer selfcalibration", IEEE Trans. Microwave Theory Tech., 39, 1991, 724-731.

[5] Gregory A.P. et Clarke R.N., "Tables of the complex permittivity of dielectric reference liquids at Frequencies up to 5 GHz", NPL report, MAT 23, 2009.

[6] Gregory A.P. et Clarke R.N., "Dielectric metrology with coaxial sensors", Measur. Sci. Technol., 18, 5, 2007, 1372-1386. 